with a diameter of $2.5 \mathrm{~nm}$ onto the sensor (see their Fig. 3 on page 448). As expected, the position of the added mass on the bridge influenced the resulting frequency change, with the change being largest for particles landing at the centre of the bridge - where the amplitude of the oscillations is the largest - and zero at the ends. By comparing the distribution of frequency changes measured in this way with the distribution expected for particles from a monodisperse source, it was possible to derive the average mass of the gold nanoparticles.

Next, they carried out mechanical mass spectrometry on a nominally pure solution of protein bovine albumin using the modelling and fitting approach described above. From only 578 binding events (resonant frequency jumps) they were able to generate a spectrum showing that the sample consists of monomers, dimers, trimers and pentamers, and to calculate the ratio between them. These measurements clearly demonstrate that it is possible to register the binding events of individual molecules. Moreover, because the molecules do not have to be ionized, this approach allows mass spectrometry to be performed on species that are difficult or even impossible to ionize.
In the future the system could be improved by making use of existing experimental techniques for decoupling mass and position ${ }^{6}$. Moreover, by miniaturizing the sample injection system, the sample volume could be made as small as a single cell. Indeed, there seem to be no technological obstacles to achieving dynamic ranges and mass resolutions better than those that are possible with conventional mass spectrometry.

One challenge is to ensure that the molecules are captured on the surface of the sensor and not lost to the sidewalls of the chamber or elsewhere. Using twodimensional arrays containing thousands of nanoscale bridges that can capture the individual binding events in a highly parallel approach would be an elegant solution to this problem. Such systems are currently being developed by a collaboration between the Caltech group and LETI in France ${ }^{7}$, and the Millipede project at $\mathrm{IBM}^{8}$ has clearly demonstrated that the parallel operation of hundreds of nanomechanical sensors and actuators is indeed possible. Moreover, the use of piezoresistive rather than magnetomotive readout would remove the need for large magnetic fields, allowing the devices to be made much smaller and more portable.
Many nanomechanical biosensors rely on the use of specific coatings on the resonators to make them sensitive to particular biomolecules. But it can be difficult to obtain reproducible results from such sensors because of device-todevice variations in the coatings. In the NEMS mass spectrometer, in contrast, the resonator does not need to be functionalized to be able to detect a given biomolecule - another great advantage of this small but mighty system.

Anja Boisen is in the Department of Micro- and Nanotechnology, Technical University of Denmark, DK-2800 Kgs. Lyngby, Denmark.

e-mail:Anja.Boisen@nanotech.dtu.dk

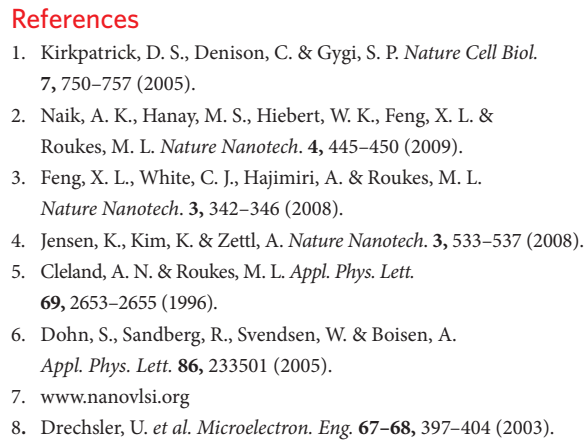

\title{
FERROELECTRIC MEMORY
}

\section{Slim fast}

Information can be stored in the polarization state of a ferroelectric material. At present, ferroelectric memories are read with a capacitive scheme that destroys the information being read. A resistive readout scheme would be nondestructive, and also faster and simpler to implement, but this approach requires thin ferroelectric layers - which are a challenge to manufacture because ferroelectricity becomes weaker as films become thinner.

Now, Manuel Bibes and colleagues have demonstrated resistive readout in ferroelectric films of $\mathrm{BaTiO}_{3}$ as thin as $1 \mathrm{~nm}$. The researchers - from the CNRS, Thales, the University of ParisSud, Cambridge University and the University of Evry - used conductive-tip atomic force microscopy (CTAFM) to observe the polarization dependence of the tunnelling current across their films. The current varied by $200 \%$ for $1-n m$ films and by $75,000 \%$ for 3 -nm films (Nature 460, 81-84; 2009).
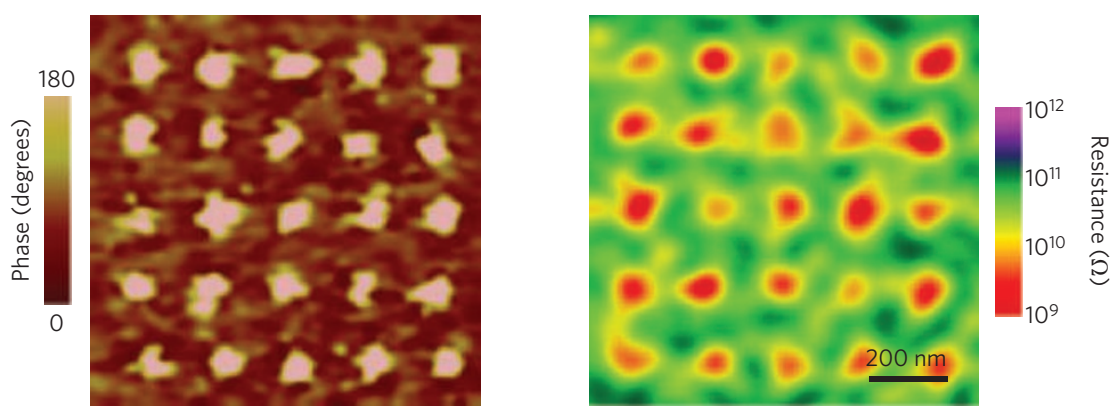

Furthermore, Bibes and co-workers were able to write to, and read from, a matrix of 70-nm sized dots. These are pictured in piezoresponse force microscopy phase images (left) and CTAFM resistance maps (right). The centres of the dots are about $200 \mathrm{~nm}$ apart, corresponding to a storage density of 16 Gbit inch $^{-2}$.

In addition to its relevance to nextgeneration ferroelectric random access memories, the work sheds light on why polarization affects the tunnelling current. The exponential dependence of the current on the thickness of the tunnelling barrier suggests an origin related to physical properties of the barrier, rather than the density of states, as has been suggested previously.

MICHAEL SEGAL 